\title{
THE RATIONALITY OF THE MODULI SPACES OF TRIGONAL CURVES OF ODD GENUS
}

\author{
SHOUHEI MA
}

\begin{abstract}
Aвstract. The moduli spaces of trigonal curves of odd genus $g \geq 5$ are proven to be rational.
\end{abstract}

\section{INTRODUCTION}

The object of this article is to prove the following.

Theorem 1.1. The moduli space $\mathcal{T}_{g}$ of trigonal curves of genus $g=2 n+1$ with $n \geq 2$ is rational.

By a trigonal curve we mean an irreducible smooth projective curve which admits a degree 3 morphism to $\mathbb{P}^{1}$. A trigonal curve of genus $g \geq 5$ has a unique $g_{3}^{1}$, so that the space $\mathcal{T}_{g}$ to be studied is regarded as a sublocus of $\mathcal{M}_{g}$, the moduli space of curves of genus $g$. Shepherd-Barron [5] proved the rationality of $\mathcal{T}_{g}$ for $g=4 n+2$ with $n \geq 1$. Hence the space $\mathcal{T}_{g}$ is rational possibly except when the genus $g$ is divisible by 4 . For the one lower gonality, Katsylo and Bogomolov [4], [1] established the rationality of the moduli spaces of hyperelliptic curves.

The proof of Theorem 1.1 is based on the classical relation between trigonal curves and the Hirzebruch surfaces $\mathbb{F}_{N}=\mathbb{P}\left(O_{\mathbb{P}^{1}} \oplus O_{\mathbb{P}^{1}}(N)\right)$. Recall that a canonically embedded trigonal curve $C \subset \mathbb{P}^{g-1}$ of genus $g \geq 5$ lies on a unique rational normal scroll $S$. The scroll $S$ may obtained either as the intersection of quadrics containing $C$, or as the scroll swept out by the lines spanned by the fibers of the trigonal map. The surface $S$ is the image of a Hirzebruch surface $\mathbb{F}_{N}$ by a linear system $\left|O_{\pi}(1) \otimes \pi^{*} O_{\mathbb{P}^{1}}(a)\right|, a>0$, where $\pi: \mathbb{F}_{N} \rightarrow \mathbb{P}^{1}$ is the natural projection. The trigonal map of $C$ is the restriction of $\pi$. When $C$ is general in the moduli $\mathcal{T}_{g}$, we have $N=0$ or 1 depending on whether $g$ is even or odd. Thus, if $L_{3, b}$ denotes the line bundle $O_{\pi}(3) \otimes \pi^{*} O_{\mathbb{P}^{1}}(b)$ on $\mathbb{F}_{1}$ with $g=2 b+1$, we have the birational equivalence

$$
\mathcal{T}_{g} \sim\left|L_{3, b}\right| / \operatorname{Aut}\left(\mathbb{F}_{1}\right)
$$

2000 Mathematics Subject Classification. Primary 14H10, Secondary 14H45.

Key words and phrases. trigonal curve, rationality of moduli. 
Here $\left|L_{3, b}\right| / \operatorname{Aut}\left(\mathbb{F}_{1}\right)$ stands for a rational quotient of the linear system $\left|L_{3, b}\right|$ by the algebraic group $\operatorname{Aut}\left(\mathbb{F}_{1}\right)$. Then Theorem 1.1 is equivalent to the following assertion in invariant theory.

Theorem 1.2. For the line bundle $L_{3, b}$ on the Hirzebruch surface $\mathbb{F}_{1}$ the quotient $\left|L_{3, b}\right| / \operatorname{Aut}\left(\mathbb{F}_{1}\right)$ is rational for $b \geq 2$.

The rest of this article is devoted to the proof of this theorem. In Section 2 we construct an $\operatorname{Aut}\left(\mathbb{F}_{1}\right)$-equivariant map from $\left|L_{3, b}\right|$ to $S^{b} \mathbb{F}_{1}$, the symmetric product of $\mathbb{F}_{1}$, which plays crucial role in the proof. In Section 3 the rationality for $g \geq 9$ is established by using the rational normal curves. In Section 4 the rationality of $\mathcal{T}_{7}$ and $\mathcal{T}_{5}$ is proved,

Throughout this article we work over the field of complex numbers. We denote by $\pi: \mathbb{F}_{1} \rightarrow \mathbb{P}^{1}$ the natural projection. The $(-1)$-curve on $\mathbb{F}_{1}$ is denoted by $\Sigma$. The line bundle $O_{\pi}(a) \otimes \pi^{*} O_{\mathbb{P} 1}(b)$ on $\mathbb{F}_{1}$ will be written as $L_{a, b}$. The bundle $O_{\pi}(1)$ is the pullback of $O_{\mathbb{P}^{2}}(1)$ by the blow-down $\mathbb{F}_{1} \rightarrow \mathbb{P}^{2}$.

\section{Symmetric product of The Hirzebruch Surface}

Let $\mathbb{P} \mathcal{E}$ be the projective space bundle $\mathbb{P} \pi_{*} O_{\pi}(2)$ on $\mathbb{P}^{1}$. The variety $\mathbb{P} \mathcal{E}$ parametrizes unordered pairs $q_{+}+q_{-}$of two points of $\mathbb{F}_{1}$ which lie on the same $\pi$-fiber. We have a rational map

$$
\varphi_{1}:\left|L_{3, b}\right| \rightarrow S^{b}(\mathbb{P} \mathcal{E}), \quad C \mapsto \sum_{i=1}^{b}\left(q_{i+}+q_{i-}\right)
$$

defined as follows. If $\left.C\right|_{\Sigma}=p_{1}+\cdots+p_{b}$ and $F_{i}$ is the $\pi$-fiber passing $p_{i}$, we set $q_{i+}+q_{i-}=\left.C\right|_{F_{i}}-p_{i}$. The map $\varphi_{1}$ is clearly Aut $\left(\mathbb{F}_{1}\right)$-equivariant. Next we define a rational map

$$
\varphi_{2}: S^{b}(\mathbb{P} \mathcal{E}) \rightarrow S^{b} \mathbb{F}_{1}, \quad \sum_{i=1}^{b}\left(q_{i+}+q_{i-}\right) \mapsto \sum_{i=1}^{b} q_{i}
$$

as follows. If $F_{i}$ is the $\pi$-fiber passing $\left\{q_{i+}, q_{i-}\right\}$ and $p_{i}=F_{i} \cap \Sigma$, there exists a unique involution $\iota_{i}$ of $F_{i} \simeq \mathbb{P}^{1}$ which fixes $p_{i}$ and interchanges $q_{i+}$ and $q_{i-}$. Then we let $q_{i} \in F_{i}$ be the fixed point of $\iota_{i}$ other than $p_{i}$. By the uniqueness of $\iota_{i}$ the map $\varphi_{2}$ is $\operatorname{Aut}\left(\mathbb{F}_{1}\right)$-equivariant. We study the composition map

$$
\varphi=\varphi_{2} \circ \varphi_{1}:\left|L_{3, b}\right| \rightarrow S^{b} \mathbb{F}_{1} \text {. }
$$

Lemma 2.1. The map $\varphi$ is dominant with a general fiber being an open set of a linear subspace of $\left|L_{3, b}\right|$.

Proof. For a general point $q_{1}+\cdots+q_{b} \in S^{b} \mathbb{F}_{1}$ let $F_{i}$ be the $\pi$-fiber passing $q_{i}$ and let $p_{i}=F_{i} \cap \Sigma$. We take an inhomogeneous coordinate $x_{i}$ of $F_{i} \simeq \mathbb{P}^{1}$ in which $p_{i}$ is $\left\{x_{i}=0\right\}$ and $q_{i}$ is $\left\{x_{i}=\infty\right\}$. The involution of $F_{i}$ fixing $p_{i}$ and $q_{i}$ is given by $x_{i} \mapsto-x_{i}$. A smooth curve $C \in\left|L_{3, b}\right|$ is contained in 
$\varphi^{-1}\left(q_{1}+\cdots+q_{b}\right)$ if and only if $\left.C\right|_{F_{i}}$ has the equation $x_{i}\left(\alpha_{i} x_{i}^{2}+\beta_{i}\right)=0$ for each $i=1, \cdots, b$. Since these are $2 b$ linear conditions on $\left|L_{3, b}\right|$, namely the vanishing of the coefficient of $x_{i}^{2}$ and the constant term for $\left.C\right|_{F_{i}}$, the second assertion is proved. The dominancy of $\varphi$ is a consequence of the dimension counting $\operatorname{dim}\left|L_{3, b}\right|=4 b+9>2 b$.

Lemma 2.2. The group $\operatorname{Aut}\left(\mathbb{F}_{1}\right)$ acts on $S^{b} \mathbb{F}_{1}$ almost freely if $b \geq 4$.

Proof. First we treat the case $b \geq 5$. If a general point $p_{1}+\cdots+p_{b} \in S^{b} \mathbb{F}_{1}$ is fixed by a $g \in \operatorname{Aut}\left(\mathbb{F}_{1}\right)$, then $g$ stabilizes a general $b \geq 5$ point set of the $(-1)$-curve $\Sigma$ so that $g$ acts trivially on $\Sigma$. Hence $g$ fixes each $p_{i}$. As $\operatorname{Aut}\left(\mathbb{F}_{1}\right)$ acts almost freely on $\left(\mathbb{F}_{1}\right)^{b}$, it follows that $g=\mathrm{id}$.

Next we study the case $b=4$. Let $f: \mathbb{F}_{1} \rightarrow \mathbb{P}^{2}$ be the blow-down. For a general $p_{1}+\cdots+p_{4} \in S^{4} \mathbb{F}_{1}$ there exists a unique smooth conic $Q$ passing $f(\Sigma)$ and $f\left(p_{1}\right), \cdots, f\left(p_{4}\right)$. Any $g \in \operatorname{Aut}\left(\mathbb{F}_{1}\right)$ fixing $p_{1}+\cdots+$ $p_{4}$, regarded as an element of $\mathrm{PGL}_{3}$, preserves $Q$ and the five point set $f(\Sigma), f\left(p_{1}\right), \cdots, f\left(p_{4}\right)$ on it. Hence $g$ acts trivially on $Q$, which implies that $g=\mathrm{id}$.

We shall apply the no-name lemma (see [3], and also [2] for nonreductive groups) to the map $\varphi$ when $b \geq 4$. For that we note the following.

Lemma 2.3. Every line bundle on $\mathbb{F}_{1}$ admits an $\operatorname{Aut}\left(\mathbb{F}_{1}\right)$-linearization.

Proof. We have canonical Aut $\left(\mathbb{F}_{1}\right)$-linearizations on the bundles $K_{\mathbb{F}_{1}}=$ $L_{-2,-1}, \pi^{*} K_{\mathbb{P}^{1}}=L_{0,-2}$, and $f^{*} K_{\mathbb{P}^{2}}=L_{-3,0}$ where $f: \mathbb{F}_{1} \rightarrow \mathbb{P}^{2}$ is the blowdown of $\Sigma$. These induce Aut $\left(\mathbb{F}_{1}\right)$-linearizations on $L_{1,0}$ and $L_{0,1}$. Since $\operatorname{Pic}\left(\mathbb{F}_{1}\right)$ is freely generated by $L_{1,0}$ and $L_{0,1}$, the lemma is proved.

By Lemma 2.3 the $\operatorname{Aut}\left(\mathbb{F}_{1}\right)$-action on $\left|L_{3, b}\right|$ is induced by an $\operatorname{Aut}\left(\mathbb{F}_{1}\right)$ representation on $H^{0}\left(L_{3, b}\right)$. Then Lemma 2.1 shows that $\left|L_{3, b}\right|$ is $\operatorname{Aut}\left(\mathbb{F}_{1}\right)$ birational to the projectivization of an $\operatorname{Aut}\left(\mathbb{F}_{1}\right)$-linearized vector bundle on an open set of $S^{b} \mathbb{E}_{1}$. By Lemma 2.2 we may apply the no-name lemma to see the

Proposition 2.4. For $b \geq 4$ we have a birational equivalence

$$
\left|L_{3, b}\right| / \operatorname{Aut}\left(\mathbb{F}_{1}\right) \sim \mathbb{P}^{2 b+9} \times\left(S^{b} \mathbb{F}_{1} / \operatorname{Aut}\left(\mathbb{F}_{1}\right)\right) .
$$

Thus the rationality of $\left|L_{3, b}\right| / \operatorname{Aut}\left(\mathbb{F}_{1}\right)$ for $b \geq 4$ is reduced to a stable rationality of $S^{b} \mathbb{F}_{1} / \operatorname{Aut}\left(\mathbb{F}_{1}\right)$.

\section{Projection of Rational normal curve}

In this section we prove a stable rationality of the quotient $S^{b} \mathbb{F}_{1} / \operatorname{Aut}\left(\mathbb{F}_{1}\right)$ to derive Theorem 1.2 for $b \geq 4$. For an integer $d \geq 0$ we consider the universal curve $f: \mathcal{H}_{d} \rightarrow\left|L_{1, d}\right|$ over the linear system $\left|L_{1, d}\right|$. The variety $\mathcal{H}_{d}$ 
is defined as a divisor on $\mathbb{F}_{1} \times\left|L_{1, d}\right|$, and $f$ is the restriction of the second projection $\mathbb{F}_{1} \times\left|L_{1, d}\right| \rightarrow\left|L_{1, d}\right|$. The bundle $L_{0,1}$ on $\mathbb{F}_{1}$ induces a relative hyperplane bundle for $f$ which we denote by $O_{f}(1)$. Let

$$
\mathcal{H}_{d, b}=\mathbb{P} f_{*} O_{f}(b) .
$$

An open set of $\mathcal{H}_{d, b}$ parametrizes pairs $\left(H, q_{1}+\cdots+q_{b}\right)$ where $H \in\left|L_{1, d}\right|$ is smooth and $q_{1}, \cdots, q_{b}$ are $b$ points on $H$. Note that a smooth $H \in\left|L_{1, d}\right|$ is a section of $\pi$.

Lemma 3.1. For $4 \leq b \leq 2 d+2$ we have a birational equivalence

$$
\mathcal{H}_{d, b} / \operatorname{Aut}\left(\mathbb{F}_{1}\right) \sim \mathbb{P}^{2 d+2-b} \times\left(S^{b} \mathbb{F}_{1} / \operatorname{Aut}\left(\mathbb{F}_{1}\right)\right) .
$$

Proof. Consider the evaluation map

$$
\psi: \mathcal{H}_{d, b} \rightarrow S^{b} \mathbb{F}_{1}, \quad\left(H, q_{1}+\cdots+q_{b}\right) \mapsto q_{1}+\cdots+q_{b} .
$$

The fiber $\psi^{-1}\left(q_{1}+\cdots+q_{b}\right)$ over a general $q_{1}+\cdots+q_{b}$ is an open set of the sub linear system of $\left|L_{1, d}\right|$ of curves passing $q_{1}, \cdots, q_{b}$. Since $\operatorname{dim}\left|L_{1, d}\right|=$ $2 d+2 \geq b, \psi^{-1}\left(q_{1}+\cdots+q_{b}\right)$ is non-empty and of dimension $2 d+2-b$. In particular, $\psi$ is dominant. Then we may apply the no-name lemma for $\psi$ as like the proof of Proposition 2.4 to deduce the equivalence (3.2).

By a comparison of Proposition 2.4 and Lemma 3.1, it suffices for the proof of Theorem 1.2 for $b \geq 4$ to show the rationality of $\mathcal{H}_{d, b} / \operatorname{Aut}\left(\mathbb{F}_{1}\right)$ for one $d$ in the range $b \leq 2 d+2 \leq 3 b+9$. We begin with the

Lemma 3.2. For $d \geq 5$ we have a birational equivalence

$$
\mathcal{H}_{d, b} / \operatorname{Aut}\left(\mathbb{F}_{1}\right) \sim \mathbb{P}^{b} \times\left(\left|L_{1, d}\right| / \operatorname{Aut}\left(\mathbb{F}_{1}\right)\right) .
$$

Proof. This lemma is an application of the no-name method for the fibration $\mathcal{H}_{d, b} \rightarrow\left|L_{1, d}\right|$. Since the bundle $L_{0,1}$ on $\mathbb{F}_{1}$ admits an $\operatorname{Aut}\left(\mathbb{F}_{1}\right)$-linearization, so is the bundle $O_{f}(1)$ on the universal curve $\mathcal{H}_{d}$. Hence the sheaf $f_{*} O_{f}(b)$ on $\left|L_{1, d}\right|$ is $\operatorname{Aut}\left(\mathbb{F}_{1}\right)$-linearized. It remains to check the almost freeness of the $\operatorname{Aut}\left(\mathbb{F}_{1}\right)$-action on $\left|L_{1, d}\right|$ for $d \geq 5$. For a general $H \in\left|L_{1, d}\right|$ the intersection $H \cap \Sigma$ is a general $d$ point set of $H \simeq \mathbb{P}^{1}$. If a $g \in \operatorname{Aut}\left(\mathbb{F}_{1}\right)$ stabilizes $H$, then we have $g(H \cap \Sigma)=H \cap \Sigma$ so that $g$ acts trivially on $H$. This is enough for concluding that $g=\mathrm{id}$.

Blowing-down $\mathbb{F}_{1}$ to $\mathbb{P}^{2}$, we see that the quotient $\left|L_{1, d}\right| / \operatorname{Aut}\left(\mathbb{F}_{1}\right)$ is birational to the $\mathrm{PGL}_{3}$-quotient of the space $\mathcal{X}_{d}$ of rational plane curves of degree $d+1$ having an ordinary $d$-fold point. Let $\widetilde{X}_{d}$ be the space of morphisms $\phi: \mathbb{P}^{1} \rightarrow \mathbb{P}^{2}$ such that $\phi^{*} O_{\mathbb{P}^{2}}(1) \simeq \mathcal{O}_{\mathbb{P}^{1}}(d+1)$ and $\phi\left(\mathbb{P}^{1}\right) \in \mathcal{X}_{d}$. We have

$$
\left|L_{1, d}\right| / \operatorname{Aut}\left(\mathbb{F}_{1}\right) \sim \mathrm{PGL}_{2} \backslash \widetilde{X}_{d} / \mathrm{PGL}_{3} \text {. }
$$

Let $\mathbb{P} V_{d+1}=\left|O_{\mathbb{P}}(d+1)\right|^{\vee}$ and $\Gamma_{d+1} \subset \mathbb{P} V_{d+1}$ be the rational normal curve $\phi_{0}\left(\mathbb{P}^{1}\right)$ where $\phi_{0}$ is the embedding associated to $O_{\mathbb{P}^{1}}(d+1)$. Recall that every 
morphism $\phi: \mathbb{P}^{1} \rightarrow \mathbb{P}^{2}$ with $\phi^{*} O_{\mathbb{P}^{2}}(1) \simeq \mathcal{O}_{\mathbb{P}^{1}}(d+1)$ is the composition of (1) the isomorphism $\phi_{0}: \mathbb{P}^{1} \rightarrow \Gamma_{d+1}$, (2) the projection $\Gamma_{d+1} \rightarrow \mathbb{P}\left(V_{d+1} / W\right)$ from a $(d-2)$-plane $\mathbb{P} W \subset \mathbb{P} V_{d+1}$ which is disjoint from $\Gamma_{d+1}$, and (3) an isomorphism $\mathbb{P}\left(V_{d+1} / W\right) \rightarrow \mathbb{P}^{2}$. The group $\mathrm{PGL}_{3}$ acts on $\widetilde{\mathcal{X}}_{d}$ by transformation of an isomorphism $\mathbb{P}\left(V_{d+1} / W\right) \rightarrow \mathbb{P}^{2}$. Hence the quotient $\widetilde{\mathcal{X}}_{d} / \mathrm{PGL}_{3}$ is naturally birational to the locus $\mathcal{Y}_{d}$ in the Grassmannian $\mathbf{G}\left(d-2, \mathbb{P} V_{d+1}\right)$ consisting of $(d-2)$-planes $\mathbb{P} W$ such that (i) $\mathbb{P} W \cap \Gamma_{d+1}=\emptyset$ and (ii) there exists a $(d-1)$-plane $\mathbb{P} U$ containing $\mathbb{P} W$ with $\mathbb{P} U \cap \Gamma_{d+1}$ being a $d$ point set. For such a $\mathbb{P} W$, the $(d-1)$-plane $\mathbb{P} U$ is spanned by the point set $\mathbb{P} U \cap \Gamma_{d+1}$ because of the fact that any distinct $d$ points on a rational normal curve in $\mathbb{P}^{d+1}$ are linearly independent. Also $\mathbb{P} U$ is uniquely determined by $\mathbb{P} W$ for an irreducible plane curve of degree $d+1$ has at most one singularity of multiplicity $d$. These two facts imply that $\mathcal{Y}_{d}$ is identified with an open set of the locus

$$
\mathcal{Z}_{d} \subset \mathbf{G}\left(d-2, \mathbb{P} V_{d+1}\right) \times\left|O_{\mathbb{P}^{1}}(d)\right|
$$

of pairs $(\mathbb{P} W, \mathbf{p})$ such that $\mathbf{p}=p_{1}+\cdots+p_{d}$ is a distinct $d$ point set on $\mathbb{P}^{1}$ and $\mathbb{P} W$ is a hyperplane of the $(d-1)$-plane $\mathbb{P} U_{\mathbf{p}}=\left\langle\phi_{0}\left(p_{1}\right), \cdots, \phi_{0}\left(p_{d}\right)\right\rangle$. We arrived at the birational equivalence

$$
\mathcal{X}_{d} / \mathrm{PGL}_{3} \sim \mathcal{Z}_{d} / \mathrm{PGL}_{2} \text {. }
$$

Now we prove the

Proposition 3.3. If $d \geq 5$ is odd, the $\mathrm{PGL}_{2}$-quotient of $\mathcal{Z}_{d}$ is rational. Hence $\left|L_{1, d}\right| / \operatorname{Aut}\left(\mathbb{F}_{1}\right)$ is rational too.

Proof. The morphism

$$
\mathcal{Z}_{d} \rightarrow\left|O_{\mathbb{P}^{1}}(d)\right|, \quad(\mathbb{P} W, \mathbf{p}) \mapsto \mathbf{p}
$$

is dominant with the fiber over a general $\mathbf{p}$ being $\mathbb{P} U_{\mathbf{p}}^{\vee}$. The vector space $U_{\mathbf{p}}$ is a subspace of $V_{d+1}=H^{0}\left(O_{\mathbb{P}^{1}}(d+1)\right)^{\vee}$. Since $d+1$ is even, the bundle $O_{\mathbb{P}^{1}}(d+1)$ is $\mathrm{PGL}_{2}$-linearized so that the $\mathrm{PGL}_{2}$-action on $\mathbb{P} V_{d+1}$ is induced by a $\mathrm{PGL}_{2}$-representation on $V_{d+1}$. Therefore $\mathcal{Z}_{d}$ is $\mathrm{PGL}_{2}$-isomorphic to the projectivization of a $\mathrm{PGL}_{2}$-linearized vector bundle on an open set of $\left|O_{\mathbb{P}^{1}}(d)\right|$. As PGL 2 acts almost freely on $\left|O_{\mathbb{P}^{1}}(d)\right|$, the no-name method applied to the fibration (3.8) shows that

$$
\mathcal{Z}_{d} / \mathrm{PGL}_{2} \sim \mathbb{P}^{d-1} \times\left(\left|\mathcal{O}_{\mathbb{P}^{1}}(d)\right| / \mathrm{PGL}_{2}\right) .
$$

The quotient $\left|O_{\mathbb{P}^{1}}(d)\right| / \mathrm{PGL}_{2}$ is rational by Katsylo [4].

Proof of Theorem 1.2 for $b \geq 4$. We may take an odd $d \geq 5$ in the range $b \leq 2 d+2 \leq 3 b+9$. By Proposition 2.4, Lemma 3.1, and Lemma 3.2 we have

$$
\left|L_{3, b}\right| / \operatorname{Aut}\left(\mathbb{F}_{1}\right) \sim \mathbb{P}^{4 b+7-2 d} \times\left(\left|L_{1, d}\right| / \operatorname{Aut}\left(\mathbb{F}_{1}\right)\right) .
$$


Then $\left|L_{1, d}\right| / \operatorname{Aut}\left(\mathbb{F}_{1}\right)$ is rational by Proposition 3.3 .

\section{THE CASE $g \leq 7$}

4.1. The rationality of $\mathcal{T}_{7}$. We consider the $\operatorname{Aut}\left(\mathbb{F}_{1}\right)$-equivariant map $\varphi$ : $\left|L_{3,3}\right| \rightarrow S^{3} \mathbb{F}_{1}$ defined in $(2.3)$. The group Aut $\left(\mathbb{F}_{1}\right)$ acts almost transitively on $S^{3} \mathbb{F}_{1}$, with the stabilizer $G$ of a general point $q_{1}+q_{2}+q_{3}$ being isomorphic to $\widetilde{S}_{3}$ by the permutation action on the set $\left\{q_{1}, q_{2}, q_{3}\right\}$. As proved in Lemma 2.1, the fiber $\varphi^{-1}\left(q_{1}+q_{2}+q_{3}\right)$ is an open set of a sub linear system $\mathbb{P} V \subset\left|L_{3,3}\right|$. Then by the slice method (see [3] ) we have the birational equivalence

$$
\left|L_{3,3}\right| / \operatorname{Aut}\left(\mathbb{F}_{1}\right) \sim \mathbb{P} V / G \text {. }
$$

The $G$-action on $\mathbb{P} V$ is induced by a $G$-representation on $V$ because the bundle $L_{3,3}$ admits an $\operatorname{Aut}\left(\mathbb{F}_{1}\right)$-linearization. It is well-known that for any linear representation $V^{\prime}$ of $\mathfrak{S}_{3}$ the quotient $\mathbb{P} V^{\prime} / \mathfrak{S}_{3}$ is rational (apply the noname method for the irreducible decomposition). Hence the quotient $\mathbb{P} V / G$ is rational, and Theorem 1.2 is proved for $b=3$.

4.2. The rationality of $\mathcal{T}_{5}$. We consider the $\operatorname{Aut}\left(\mathbb{F}_{1}\right)$-equivariant map $\varphi_{1}$ : $\left|L_{3,2}\right| \rightarrow S^{2}(\mathbb{P} \mathcal{E})$ defined in (2.1).

Lemma 4.1. The group Aut $\left(\mathbb{F}_{1}\right)$ acts almost transitively on $S^{2}(\mathbb{P} \mathcal{E})$ with the stabilizer $G$ of a general point $\mathbf{q}=\left(q_{1+}+q_{1-}\right)+\left(q_{2+}+q_{2-}\right)$ being isomorphic to $\widetilde{\Xi}_{2} \ltimes\left(\mathfrak{\Xi}_{2} \times \widetilde{\Xi}_{2}\right)$.

Proof. Since $\operatorname{Aut}\left(\mathbb{F}_{1}\right)$ and $S^{2}(\mathbb{P} \mathcal{E})$ have the same dimention, it suffices to calculate the stabilizer $G$. If $p_{i \pm} \in \mathbb{P}^{2}$ is the image of $q_{i \pm}$ by the blow-down $\mathbb{F}_{1} \rightarrow \mathbb{P}^{2}$, the group $G$ is identified with the group of those $g \in \mathrm{PGL}_{3}$ such that for each $i=1,2$ we have $g\left(\left\{p_{i_{+}}, p_{i_{-}}\right\}\right)=\left\{p_{j_{+}}, p_{j_{-}}\right\}$for some $1 \leq j \leq$ 2.

Let $F_{i}$ be the $\pi$-fiber passing $q_{i \pm}$ and let $p_{i}=F_{i} \cap \Sigma$. The fiber $\varphi_{1}^{-1}(\mathbf{q})$ is an open set of the sub linear system $\mathbb{P} V \subset\left|L_{3,2}\right|$ of curves passing $q_{1+}, \cdots, q_{2-}$ and $p_{1}, p_{2}$. Similarly as Section 4.1, the slice method applied to the map $\varphi_{1}$ implies that

$$
\left|L_{3,2}\right| / \operatorname{Aut}\left(\mathbb{F}_{1}\right) \sim \mathbb{P} V / G
$$

where the $G$-action on $\mathbb{P} V$ is induced by a $G$-representation on $V$. Let $\mathbb{P} W \subset$ $\mathbb{P} V$ be the sub linear system defined by

$$
\mathbb{P} W=2 F_{1}+2 F_{2}+2 \Sigma+\left|L_{1,0}\right| \text {. }
$$

Since the group $G$ preserves the curves $F_{1}+F_{2}$ and $\Sigma$, the subspace $\mathbb{P} W$ is invariant under the $G$-action. Since $G$ is finite, we have a $G$-decomposition $V=W \oplus W^{\perp}$ where $W^{\perp}$ is a $G$-invariant subspace. The group $G$ acts almost 
freely on the linear system $\left|L_{1,0}\right|$. Hence we may apply the no-name lemma for the projection $\mathbb{P} V \rightarrow \mathbb{P} W$ from $\mathbb{P} W^{\perp}$ to see that

$$
\mathbb{P} V / G \sim \mathbb{C}^{9} \times(\mathbb{P} W / G) .
$$

The quotient $\mathbb{P} W / G$, being of dimension 2 , is rational by Castelnuovo's theorem. This completes the proof of rationality of $\mathcal{T}_{5}$.

\section{REFERENCES}

[1] Bogomolov, F. A.; Katsylo, P. I. Rationality of some quotient varieties. Mat. Sb. (N.S.) 126(168) (1985), 584-589.

[2] Chernousov, V.; Gille, P.; Reichstein, Z. Resolving G-torsors by abelian base extensions. J. Algebra 296 (2006), 561-581.

[3] Dolgachev, I. V. Rationality of fields of invariants. Algebraic geometry, Bowdoin, 1985, 3-16, Proc. Symp. Pure Math., 46, Part 2, Amer. Math. Soc., Providence, 1987.

[4] Katsylo, P. I. Rationality of the moduli spaces of hyperelliptic curves. Izv. Akad. Nauk SSSR. 48 (1984), 705-710.

[5] Shepherd-Barron, N. I. The rationality of certain spaces associated to trigonal curves. Algebraic geometry, Bowdoin, 1985 (Brunswick, Maine, 1985), 165-171, Proc. Symp. Pure Math., 46, Part 1, Amer. Math. Soc., Providence, RI, 1987.

Graduate School of Mathematical Sciences, the University of Tokyo, 3-8-1 Komaba, MEgUro-KU, TOKyo 153-8914, JAPAN

E-mail address: sma@ms.u-tokyo.ac.jp 